\title{
Identifying The Health Needs In Rural Appalachian Ohio: Outcomes Of A Rural Community-Academic Partnership
}

\author{
Ronald A. Vance, MSN, RN, Ohio University, Chillicothe, USA \\ Tania B. Basta, Ph.D., MPH, CHES, Ohio University, Athens, USA \\ Jennifer J. Bute, Ph.D., Indiana University-Purdue University Indianapolis, USA \\ Sharon A. Denham, RN, DSN, Ohio University, USA
}

\begin{abstract}
To identify health issues in two rural counties, a needs assessment was developed by health officials and researchers. Focus groups $(n=32)$ and interviews $(n=8)$ were conducted among community leaders and a modified BRFFS survey was completed by 399 community members. Results indicated the health of the participants was influenced by: 1) rural Appalachian culture, 2) geography and access to health care, and 3) lack of access/knowledge about preventive health behaviors. These issues likely contributed to $30 \%$ obesity prevalence among the sample, which was prioritized as the main health issue for both counties.
\end{abstract}

Keywords: Community Health Needs Assessment; Rural Health, and Community-Academic Partnership

\section{INTRODUCTION}

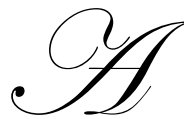

ppalachia, as defined by the Appalachian Regional Commission (ARC) is a 205,000 mile square region which includes all of West Virginia and parts of 12 other states, including Ohio (ARC, 2011). About 23 million people, or $8 \%$ of the nation, live in 420 counties in the Appalachian Region. Most communities are remote or isolated, with $42 \%$ of all individuals living in rural areas compared to $20 \%$ of the national population (ARC, 2011). Many problems persist as large pockets of rural areas lag behind much of the nation regarding levels of income, employment, educational attainment, and poverty (Wood \& Bischak, 2000).

Rural Appalachian populations tend to have higher poverty rates and health disparities, less education and literacy, less nutritional diets, and riskier health behaviors than their urban counterparts (Wewer, Katz, Fickle, \& Paskett, 2006). Evidence suggests that higher disease and mortality rates are due to social, cultural, and economic influences (Coyne, Demian-Popescu, \& Friend, 2006). Health disparities are substantiated by high mortality rates and hospitalizations throughout Appalachia for heart disease, certain types of cancer, stroke, chronic obstructive pulmonary disease, accidents, diabetes, suicide, and infant death (Halverson, Ma, \& Harner, 2009). Diets high in fat content, cigarette smoking, low socioeconomic status, and drug and alcohol addiction have increased the health risks for those living in the region (Caldwell, 2007). In addition to common health care concerns faced by the majority of Americans, such as rising health care costs, those in Appalachia have additional concerns such as inaccessibility to services, shortages of health care workers, and few culturally sensitive health care services (Blakeney, 2005; 2006). Also, many of the rural areas in Appalachia are designated as Medically Underserved Areas (MUAs), or Health Professional Service Areas (HPSAs), thus making it difficult for individuals to receive proper preventive and restorative care (Health Resources and Services Administration [HRSA], 2011).

Meigs and Vinton Counties are two rural, medically underserved counties in Appalachian Ohio. They are two of the least populated counties in the state of Ohio, with 22,838 and 13,288 citizens respectively (Ohio Department of Jobs and Family Services [ODJFS], 2011). In July 2011, Meigs County had the second highest unemployment rate in the state of Ohio at just over 14\% while Vinton had a rate at almost 12\%: both higher than the state and national averages (ODJFS, 2011). Furthermore, the 2009 median household income was $\$ 34,359$ in Meigs 
County and $\$ 34,275$ in Vinton County compared to $\$ 45,467$ for the rest of Ohio, and nearly $20 \%$ of the households in both counties are below the poverty line (U.S. Census Bureau, 2011). While demographic information is easily accessible, health status and health behavior information is not readily available for these two counties, which makes it difficult to prioritize local health needs.

Therefore, in an effort to address the aforementioned issues, the health department officials in both counties were approached by the Director of the Appalachian Rural Health Institute (ARHI) at Ohio University about partnering to identify ways to positively affect the health status of the underserved citizens in their communities. It was mutually decided that community input was needed in order to identify and prioritize the health needs of Meigs and Vinton counties as well as to gather data that could be used for grant writing purposes. Agreement was reached for university researchers and community persons to collaborate in conducting a needs assessment.

\section{METHODS}

\section{Research Design}

A mixed-methods design was employed in which cross-sectional quantitative survey data and qualitative focus group and interview data were collected in 2008 from individuals living in Meigs and Vinton counties in Southeastern Ohio. This study was approved by the Institutional Review Board at Ohio University.

\section{Participant Recruitment and Sample}

Qualitative. Participants in both the focus group and interview sessions were recruited through a strategic, snowball sampling strategy (Patton, 2002). One researcher is a resident of Meigs County and had previously worked with members of both counties on a range of health initiatives. Local leaders from each county worked with local health leaders to identify key informants, local residents and community leaders willing to participate in an interview. When planning the interview sessions, wide community representation was sought. For example, a judge and a chamber of commerce representative from each county were included in order to gain perspective about the scope of community health issues. The resulting sample of interview participants consisted of six participants in Meigs County and two in Vinton County, with a final sample of 8 interview participants. Demographic information about the interview and focus group participants was not collected due to difficulties in maintaining anonymity in such closely-knit communities.

In designing the focus group sessions, three focus groups were organized for each county with a total of six sessions $(n=32)$. In each county one focus group session included community leaders ( $n=5$ in Meigs County; $n=8$ in Vinton County), one included health providers ( $n=3$ in Meigs County; $n=4$ in Vinton County), and one included health consumers ( $n=5$ in Meigs County; $n=7$ in Vinton County). Community leader sessions included residents who were local business leaders in their respective counties and those connected to community agencies and institutions that served a broad swath of local residents. Health provider sessions were comprised of local health officials, including representatives from county health departments, school nurses, and directors of other local health agencies. Finally, the consumer group included a wide variety of citizens who were not officially affiliated with health services or typically designated as community leaders, but offered insights about health-related issues in their county of residence.

Quantitative. Survey participants were recruited via convenience sampling in both counties with a total of 399 surveys completed. Nearly $60 \%$ of the surveys were collected from county fairs $(n=236), 21 \%$ from flu clinics $(n=$ $84), 11 \%$ at an air show $(n=44)$, and $9 \%$ at various health screenings at the county health departments $(n=34)$. Ninety-three percent of the participants self-identified as White, $68 \%$ as female, $83 \%$ as high school graduates and $51 \%$ were employed either full time or part time. The average age of the participants was $49(S D=16)$ years old. Seventy-three percent of the participants had some type of health insurance, such as private, Medicaid or Medicare. See Table 1 for more detailed participant demographic information. 
Table 1

Demographics of Community Needs Assessment Participants

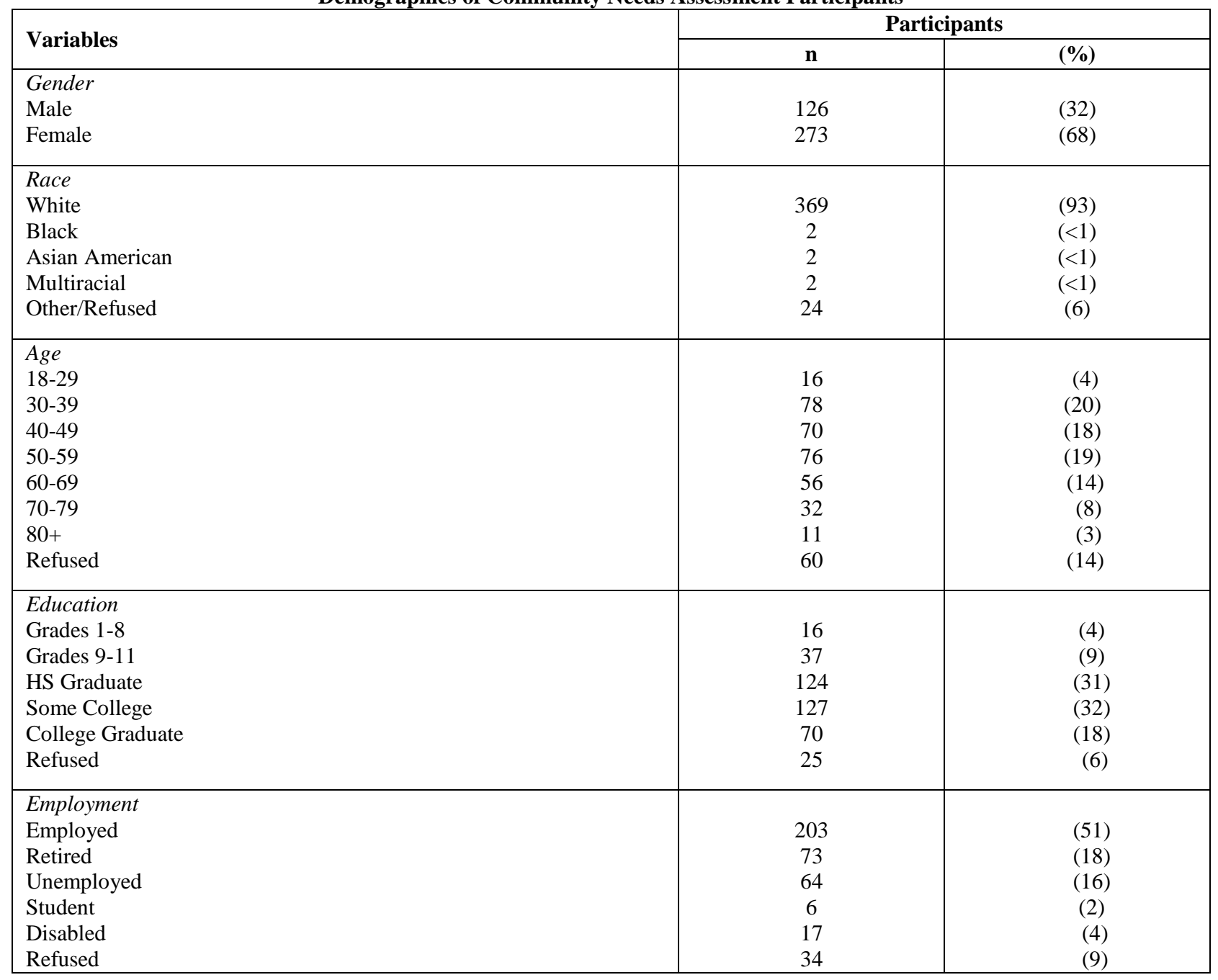

\section{Data Collection}

Qualitative. The data collection process consisted of conducting face-to-face semi-structured interviews and focus groups. The interviews lasted approximately 60 minutes per session, as did the focus groups. The fourth author moderated all interview and focus group sessions, while another author assisted as a note taker for the sessions. The researchers opted not to record the interviews or focus groups for two primary reasons. First, the researchers were aware of local cultural norms that can promote suspicion of community outsiders. Therefore, they sought to build trust among participants and decided the presence of a tape recorder could put that trust at risk. Second, the researchers were aware that participants might reveal private information about family members, friends, and neighbors or might criticize the practices of the agencies that employed them. So, they sought to ensure an atmosphere of trust and promote less inhibited disclosure. In lieu of recording the sessions and creating transcripts, copious notes were taken at each session.

Quantitative. Four hundred surveys were distributed in 2008 to individuals at flu clinics at the local health departments, county fairs, and an air show. Local public health practitioners in both counties distributed and collected the questionnaires from the participants who attended the flu clinics, and university researchers and local leaders at the other community events. 


\section{Instruments}

Qualitative. The questions in both the focus group and interview sessions elicited responses about community and health-related topics. More specifically, participants were asked to share their insights about (a) health concerns and problems in the community, (b) health resources available in the county, (c) potential barriers to good health, (d) perceptions of problematic health behaviors, (e) initiatives residents take to improve their health, (f) special health concerns for participants or their families, and (g) ideas for promoting individual and community involvement in health efforts.

Quantitative. A 37-item modified Behavioral Risk Factor Surveillance Survey (BRFSS) was designed to assess individual health status, risk factors for chronic disease, and health behaviors (Center for Disease Control and Prevention, 2006). The majority of the items were closed ended, but a few were open ended in order to allow for further elaboration. The 2007 BRFSS was adapted so that individuals with low literacy would be able to complete them with little or no assistance. One of the investigators has significant experience in Appalachian culture and literacy and was instrumental in designing the survey instrument.

\section{Data Analysis}

Qualitative. Researchers' notes from the interview and focus group sessions were the key data source for the qualitative portion of the study. Thematic analysis was conducted of all the session notes to support and clarify the findings in the quantitative survey (Lincoln \& Guba, 1985).

Quantitative. Descriptive statistics were used to describe the sample and present the prevalence of chronic disease, risk factors, and health behaviors. All analyses were conducted using Statistical Package for the Social Sciences (SPSS) Version 16.0.

\section{RESULTS}

\section{Qualitative}

Results indicated that the focus group and interview participants in this study expressed several issues related to the health status of local county residents. Many of the same issues were discussed by multiple participants, and they can best be conceptualized in three themes: 1) rural Appalachian culture, 2) geography and access to health care, and 3) lack of access/knowledge about preventive health behaviors. These themes were expressed to some extent among the participants in the focus groups and interviews, regardless of job type, gender, or socio-economic status.

Rural Appalachian culture. Participants in the focus group and interview sessions explained that particular aspects of Appalachian culture combined with the challenges of geographic location often prevent community members from making use of available services (e.g., free health screenings) and limit their access to adequate health care resources (e.g., emergency care, physician specialists). Residents of both counties suggested that a subculture exists that is not well understood by middle class residents or policy makers. For instance, those living in poverty in rural Appalachia are reluctant to accept help from others, particularly those outside their local community. This tendency was described by one participant as a cultural norm of being "too proud to ask for help." Indeed, many participants attributed a reluctance to take advantage of particular services to the social stigma associated with government welfare programs. Residents tend to equate any sort of free programs and services with public welfare, and thus, view participation in programs or acceptance of help as stigmatizing. As one participant said, "Free' has a stigma of being associated with welfare so people avoid taking or attending programs." As a result, some individuals and families who are eligible for services do not seek them. While local residents may be anxious to take care of their own and are willing to share with one another, many are apprehensive about outsiders and about accepting assistance. These cultural norms ultimately have great potential to inhibit health.

Moreover, participants explained that multigenerational poverty is common, making health care that is not offered at free or reduced costs unaffordable for many. Citizens that were unemployed or underemployed are often 
described as the "working poor." A number of local workers in both counties exist on minimum wage jobs that often did not provide affordable health care benefits. Participants described local families in which both parents work full time outside the home yet have no access to health insurance or are unable to pay costs such as co-pays even if they are insured. Multiple problems are overwhelming family systems, including grandparents raising children, teenagers becoming parents, and large members of extended family members living together. Some report that many children are sent to school hungry and that many youth are "couch jumpers" or homeless and move from one friend's home to another. Participants said that many individuals and families are so overwhelmed that they have no idea where or how to begin to make changes, let alone focus on preventative health behaviors. These trends of working at lowpaying jobs, inadequate access to health care, unstable living conditions, and distrust of accepting help tend to pass, in some families, from one generation to another.

Geography and access to health care. All of the aforementioned factors are compounded and exacerbated by the challenges of rural geography. Pressing concern expressed by focus group and interview participants was the need for local health care services and providers. Neither county has its own hospital and specialty providers, such as cardiologists and oncologists, are particularly hard to find. As one person noted, "Every county should at least have its own emergency room." Residents in need of long-term or specialized care are often required to travel a two to three hour car ride each way. Travelling to receive health care poses a host of problems, not the least of which is the additional financial burden associated with taking time off from and paying transportations expenses (e.g., gas, food). In addition, participants explained that many residents are uncomfortable traveling so far from home and driving in urban traffic conditions. As such, the geographic locale impedes access to health care services.

Lack of access/knowledge about prevention health behaviors. In addition to the combined forces of culture and geography, issues of low health literacy and a lack of access to the resources necessary to maintain healthy lifestyles result in diminished health outcomes. Participants, particularly those in the health provider focus group, shared their impression that most people do not understand the role and importance of preventative care. Examples often cited were things like tobacco used and the roles of activity and nutrition to prevent overweight and obesity (e.g., diabetes, heart disease). They explained that mainstream ideas about health and wellness are not valued or understood. Several of the participants stated things like: "the preventative education piece is missing," "prevention isn't important," and some suggested that health officials and providers must cope with a "fix it after it happens culture." In addition, participants observed that most local residents do not participate in health related programs, which could result from a lack of motivation or awareness of all that is available. Many residents lack skills needed to alter their current way of life and the solutions offered by outsiders seldom appear as viable or reasonable solutions to those in the throes of poverty.

Even those who desire to make positive health changes and engage in healthy diet and exercise behaviors may have difficulty doing so. Participants showed great concern about the limited options for grocery shopping. In Vinton County, only a single grocery store is available, with smaller local markets linked to gas stations or Dollar Stores. Residents in Meigs County have a few more options, but costs may be higher for food items and choices limited. Thus, people often purchase less expensive processed or microwaveable foods, consume products such as potatoes and bologna, which are cheaper, or eat fast food when the option is available. Local health professionals questioned whether parents had adequate knowledge about cooking, nutrition, and budget management. Cooking was described as a "lost art" for many in the area. While farming, local gardens, and food preservation were once commonplace, most individuals are not using affordable approaches, such as home gardens, to grow more nutritious and affordable fruits and vegetables. Participants indicated it is not uncommon for local food pantries to be supporting two or three generations of related family members because multigenerational poverty is so common.

Participants also expressed concern about what they described as a "lack of physical and extracurricular activities." Few facilities are fully used by local residents to exercise. Although natural resources in Vinton County include 40,000 acres of public land (i.e., Zaleski State Forest, Wayne National Forest, Tar Hollow, Raccoon Management Area, Lake Hope, Lake Rupert, Lake Alma ), they are underused by local residents. While the local high school has a fitness center, many rural residents do not feel welcome there, and no public gyms or health programs currently exist in the county. The local senior center in Meigs County is quite active and has a fitness center available. In the last few years walking paths in Meigs have been created in the towns of Pomeroy, Racine, and, Middleport. Yet, many local residents believe there is little in these communities for youth and families to do. Widespread boredom is reported, and, while not the only reason, many turn to alcohol and substance abuse. 


\section{Quantitative}

Overall perceived health. Approximately two-thirds of the participants described their overall health status as good $(38 \%)$ or very good $(35 \%)$, while only $10 \%$ felt they were in excellent overall health, $14 \%$ in fair health and $3 \%$ in poor health.

Chronic disease. The most frequently reported chronic conditions, which had been diagnosed by a health care professional, were arthritis (35\%), asthma (16\%), diabetes (14\%), and coronary heart disease (10\%). From the rural health literature, it was expected there would be high prevalence of diabetes and coronary heart disease (CHD), so the following information was collected on these conditions.

Coronary heart disease. Over ten percent of the participants reported they had been told they had coronary heart disease. Of those who had been received a CHD diagnosis, $46 \%$ already experienced a heart attack and $24 \%$ had experienced a stroke or Transient Ischemic Attack (mini stroke). Furthermore, an additional 15 individuals reported they had a heart attack or a stroke, but had not been diagnosed with CHD.

Diabetes. Thirteen percent of the participants reported they had received a diabetes diagnosis (not during pregnancy) and the average age of diagnosis was 44 years $(S D=18)$. Seventy percent of the participants living with diabetes reported taking diabetes medication or insulin. However, when asked about their ability to perform routine diabetes care, $20 \%$ reported they were unable to visit a health professional on a regular basis due to the inability to pay, $43 \%$ were unable to regularly adhere to their prescribed medication due to inability to pay, $43 \%$ were unable to purchase home test strips to test their blood sugar and $20 \%$ were unable to attend a diabetes education program.

Table 2

Prevalence Data for Meigs/Vinton Counties, Ohio, and United States

\begin{tabular}{lccc}
\hline Condition/Behavior & $\begin{array}{c}\text { Meigs/Vinton } \\
\text { Counties (\%) }\end{array}$ & Ohio $(\%)^{*}$ & US (\%)* \\
\hline Arthritis & $\mathbf{3 5 . 3}$ & 32.2 & 27.5 \\
Asthma & $\mathbf{1 6 . 3}$ & 14.2 & 13.6 \\
Diabetes & $\mathbf{1 3 . 5}$ & 9.9 & 8.3 \\
CHD & $\mathbf{1 0 . 2}$ & 5.2 & 4.3 \\
Heart Attack & $\mathbf{6 . 8}$ & 4.9 & 4.2 \\
Stroke & $\mathbf{4 . 5}$ & 2.9 & 2.6 \\
High Cholesterol & $\mathbf{4 1 . 6}$ & 39.6 & 37.6 \\
Hypertension & $\mathbf{3 1 . 0}$ & 28.4 & 27.8 \\
Overweight & 28.8 & 34.1 & 36.5 \\
Obesity & $\mathbf{3 0 . 0}$ & 29.3 & 26.7 \\
Tobacco Use & 14.2 & 20.1 & 18.4 \\
Did not Meet PA Guidelines & $\mathbf{6 2 . 2}$ & 50.0 & 50.5 \\
\hline
\end{tabular}

*Data from BRFSS Data 2007 or 2008 (Some questions asked on alternate years)

Bold $=$ Higher prevalence in current sample

Risk factors for chronic disease. The most frequently reported risk factors for chronic disease, which had been diagnosed by a health care professional, were high cholesterol (42\%), hypertension (not during pregnancy) (31.0\%), overweight (29\%), and obesity (30.0\%). Among those who were diagnosed with hypertension, nearly $90 \%(n=$ 109) were currently taking prescription medication to manage the condition.

Depression. A quarter of the participants reported "feeling sad, blue, or depressed for at least two weeks in the last 12 months;" a symptom indicative of depression. Given the high proportion of individual who responded they felt depressed, we compared the data by county and found that $34 \%$ of the participants in Vinton County responded they felt depressed which was statistically higher $(z=2.50, \mathrm{p}<.05)$ than the $21 \%$ participants from Meigs County.

Health behavior: tobacco use. At one time, $40 \%$ of the participants used some form of tobacco (cigarettes and/or smokeless tobacco). However, at the time of this survey, $66 \%$ of those who reported tobacco use had quit smoking, only $14 \%$ reported regular tobacco use, and only $2 \%$ reported occasional tobacco use. 
Health behavior: physical activity. According to the BRFSS physical activity recommendations, only $38 \%$ of the participants met the requirements of 30 minutes of physical activity on 5 or more days a week or 20 minutes of vigorous activity on 3 or more days per week.

\section{DISCUSSION}

The purpose of this community health needs assessment was to identify health issues of importance to the residents in two Appalachian Ohio counties. The results of this needs assessment have been have been used to prioritize health needs as well as substantiate needs for increased funding for services. Three themes, which affect health status in these counties, emerged from the qualitative data and can be conceptualized as: 1) rural Appalachian culture, 2) geography and access to health care, and 3) lack of access/knowledge about preventive health behaviors. The quantitative results indicated that chronic disease prevalence was higher than the state and national averages, which are likely related to the issues identified in the interviews and focus groups.

The first theme highlighted the impact of rural Appalachian culture on health care and preventive services. Participants discussed that even if health services are available and accessible, many individuals living in Appalachia do not access these services due to their "reluctance to accept help from others" and the social stigma attached to free programs. While social stigma may be related to government assistance programs, some participants stated that this notion of stigma has also prevented individuals from participating in "free" preventive screenings because of the association of free as meaning "public welfare."

Another issue that was discussed was the idea of family systems being stressed and overwhelmed due to teenage pregnancy, multiple generations living in one household, food insecurity, and homelessness. The notion of children being homeless and being sent to school hungry illustrates the poverty that has become the norm for some county residents. As a result of these family stressors, many believe themselves helpless to make changes and neglect preventive health behaviors. Helplessness was illustrated by the large number of participants in Meigs and Vinton counties, respectively, who reported feeling "depressed, sad or blue most of the day, nearly every day for 2 weeks or longer." This high rate of reported symptoms indicative of depression surpasses the national rate of $10 \%$ of individuals aged 18 and older who are diagnosed with clinical depression annually (National Institutes of Mental Health [NIMH], 2010).

The second theme centered on the ways geographic location prevents rural Appalachian residents from accessing adequate health care. Participants expressed deep concern about the paucity of health care providers available within convenient driving distance. This concern is perhaps most starkly supported by the troubling rates of diabetes in this region (Serrano, Leiferman, \& Dauber, 2007; Smith \& Tessaro, 2005). In this study, 13\% of the survey respondents reported receiving a diabetes diagnosis, but many of these individuals reported an inability to visit a health care professional on a regular basis. Though lack of insurance coverage and economic hardship likely contributes to these trends it is also likely that many of these residents do not have a health care provider nearby (Smith \& Tessaro, 2005).

The third theme, lack of access/knowledge about preventive health behaviors, was mentioned repeatedly in the focus group and interviews. One of the underlying issues was related to low levels of health literacy or the "degree to which individuals have the capacity to obtain, process, and understand basic health information and services needed to make appropriate health decisions" (Health Resources and Services Administration [HRSA], 2009). Individuals, who are not health literate, may have difficulty following directions on prescription drug bottles, doctor's instructions, reading educational brochures, and applying self-care information (HRSA, 2010). Therefore, in an effort to avoid embarrassment or confusion, individuals may not take medication or delay seeking medical attention, resulting in further medical complications.

Individuals did not seem to value prevention as a priority. In fact, the health care providers who were interviewed in this study indicated that individuals prefer to "fix it after it happens" instead of preventing the problem in the first place. These behavioral factors may contribute to the higher prevalence of chronic conditions in these counties as compared to the State of Ohio and the Nation. Similarly, the results were furthered illustrated by the low percentage of participants who met physical activity guidelines for regular moderate activity $(38 \%)$. 
Another issue related to theme three was that even if individuals want to make positive health behavior changes, their environment is not supportive. For example, participants discussed the limited food options in both counties that force residents to eat fast food or less expensive processed food that can be purchase at convenience stores. This lack of access to healthy food options, including fruits and vegetables, has potential to precipitate many of the health disparities seen among individuals living in Appalachia (Kropf, M.L., Holben, D., Holcomb, J.P., \& Anderson, H., 2007; Walker, J., Holben, D.H., Kropf, M., Holcomb, J.P., \& Anderson, H., 2007). Similarly, participants expressed concern about the lack of physical activities in their communities, even though state parks are located in both counties. Therefore, the lack of physical activity coupled with the limited food choices are both contributing factors to the higher percentage of obese (30\%) individuals in these counties as compared to Ohio (29\%) and the US (26\%).

\section{LIMITATIONS}

There were some limitations in this study that should be discussed. One limitation is that the data collected is not representative of all of the residents in Meigs and Vinton Counties. Since this study received minimal funding, it was not possible to collect a truly representative sample for the quantitative portion of the needs assessment. Obviously, the qualitative data was not representative given the nature and purpose of the methodology. Therefore, results of this needs assessment should be interpreted with caution and not generalized beyond the study sample.

Another limitation is that the focus groups and interviews were not audio-recorded in this study. While this was done intentionally as not to create anxiety among the participants, certainly there may be data that was not captured as a result of not having a verbatim transcript of the discussion. However, we feel that this limitation may have also strengthened the study. We feel that we may have received a more accurate depiction of the health issues in these counties because participants did not feel threatened by being audio-taped.

\section{Needs Assessment Outcomes and Next Steps}

Despite the limitations, the quantitative results of this needs assessment were shared with health officials in both Meigs and Vinton counties in early 2009. Then, the health officials presented the results to the Family and Child First Councils (FCFC) in each county so that the council members could identify a priority health issue for each county. Obesity was selected as the health issue in both counties due to the $30 \%$ prevalence and the high number of obesity-related conditions that were identified. As a result, three federal grant applications were developed collaboratively among the researchers and the health officials. All three of the proposals were focused on using a community-based participatory (CBPR) approach to prevent and reduce the prevalence of obesity in these communities. Although the proposals were not funded, the researchers and county officials continue to collaborate in an effort to seek funding to prevent and reverse chronic disease in Appalachia. Finally, to highlight the needs of individuals living in Appalachia, these needs assessment results were presented at two national conferences in 2010.

\section{AUTHOR INFORMATION}

Ronald A. Vance, MSN, RN is an Assistant Professor of Nursing at Ohio University on the Chillicothe campus. He is currently working on his $\mathrm{PhD}$ in Cultural Studies from the College of Education. His areas of research are HIV, health issues affecting the people of Appalachia, and the utilization of technology in nursing education. E-mail: vancer1@ohio.edu. Corresponding author.

Tania B. Basta, Ph.D., MPH, CHES is an Associate Professor in the Department of Social and Public Health in the College of Health Sciences and Professions at Ohio University in Athens, Ohio. She also serves as the Associate Director of the Appalachian Rural Health Institute at Ohio University. She has extensive experience with community-based participatory research approaches and HIV-related research. E-mail: basta@ohio.edu

Jennifer J. Bute (Ph.D., University of Illinois at Urbana-Champaign) is an Assistant Professor in the Department of Communication Studies at Indiana University-Purdue University Indianapolis. She studies communication about health in interpersonal relationships and public discourse about women's health. She is particularly interested in issues related to privacy, social support, and gender. She teaches graduate and undergraduate courses in health, 
interpersonal, and gender communication. Her work has appeared in numerous edited books and journals, including Health Communication, Communication Monographs, Human Communication Research, Communication Studies, Review of Communication, Qualitative Health Research, and Social Science and Medicine. E-mail: jjbute@iupui.edu

Sharon A. Denham DSN, RN is Professor Emerita at Ohio University's School of Nursing. She is Project Director at the Center for Appalachian Philanthropy for a \$2.5 million CDC funded project called Strengthening Communities to Prevent Diabetes in Rural Appalachia, a 5-year grant aimed at diabetes prevention. Through work with the CDC National Diabetes Education Program, she developed Diabetes: A Family Matter, a culturally sensitive program and toolkit that addresses health literacy concerns. She has numerous publications from her research about Appalachia. Her Family Health Model suggests an ecological lens to 'think family' in practice. E-mail: denham@ohio.edu

\section{REFERENCES}

1. Appalachian Regional Commission. (2011). ARC Designated Distressed Counties - Fiscal Year 2009. Retrieved from http://www.arc.gov/appalachian_region/TheAppalachianRegion.asp

2. Blakeney, A.B. (2005). Educating culturally sensitive health professionals in Appalachia. In S.E. Keefe (Ed.), Appalachian Cultural Competency. Knoxville, TN: The University of Tennessee Press.

3. Blakeney, A.B. (2006). Health care in Appalachia. In G.T. Edwards, J.A. Asubury, \& R.L. Cox (Eds). A Handbook to Appalachia. Knoxville: University of Tennessee Press.

4. Caldwell, D.R. (2007) Bloodroot: Life stories of nurse practitioners in rural Appalachia. Journal of Holistic Nursing. 25(2). 73-79.

5. Centers for Disease Control and Prevention. (2006, December). Behavioral risk factor surveillance system questionnaire. Retrieved from http://www.cdc.gov/brfss/questionnaires/pdf-ques/2007brfss.pdf

6. Coyne, C. A., Demian-Popescu, C., \& Friend, D. (2006). Social and cultural factors influencing health in Southern West Virginia: A qualitative study. Prevention of Chronic Disease. 3(4) 124-126.

7. Halverson, J.A., Ma, L., \& Harner, E.J. (2004, September) An analysis of disparities in health status and access to health care in the Appalachian region. Washington, DC: The Appalachian Regional Commission. Retrieved from http://www.arc.gov/research/researchreportdetails.asp?REPORT ID=82

8. Health Resources and Services Administration. (2011). Health Professional Shortage Areas by State and County. Retrieved from http://hpsafind.hrsa.gov/HPSASearch.aspx

9. Health Resources and Services Administration (2010). Health literacy. Retrieved from http://www.hrsa.gov/healthliteracy/

10. Kropf, M.L., Holben, D., Holcomb, J.P., \& Anderson, H. (2007). Food security status and produce intake and behaviors of special supplemental nutrition program for women, infants, and children and farmers' market nutrition program participants. Journal of the American Dietetic Association. 107(11) 1903-1908.

11. Lincoln, Y., \& Guba, E. (1985). Naturalistic inquiry. Beverly Hills, Ca: Sage.

12. National Institute of Mental Health. (2010). The numbers count: Mental disorders in America. Retrieved from http://www.nimh.nih.gov/health/publications/the-numbers-count-mental-disorders-inamerica/index.shtml\#Mood

13. Ohio Department of Jobs and Family Services. (2009). December 2009 ranking of Ohio County unemployment rates. Retrieved from http://lmi.state.oh.us/LAUS/Ranking.pdf

14. Patton, M.Q. (2002). Qualitative Research and Evaluation Methods. 3rd Ed. Thousand Oaks: Sage Publications.

15. Serrano, E., Leiferman, J., \& Dauber, S. (2007). Self-efficacy and health behaviors toward the prevention of diabetes among high risk individuals living in Appalachia. Journal of Community Health. 32(2). 121133.

16. Smith, S. L. \& Tessaro, I. A. (2005). Cultural perspectives on diabetes in an Appalachian population. American Journal of Health Behavior. 29(4) 291-301.

17. U.S. Census Bureau. (2011). Ohio quick facts. Retrieved from http://quickfacts.census.gov/qfd/states/39000.html 
18. Walker, J., Holben, D.H., Kropf, M., Holcomb, J.P., \& Anderson, H. (2007). Household food insecurity is inversely associated with social capital and health in females from Special Supplemental Nutrition Program for Women, Infants, and Children Households in Appalachian Ohio. Journal of the American Dietetics Association. 107(11). 1989-1993.

19. Wewer, M.E., Katz, M., Fickle, D., \& Paskett, E.D. (2006). Risky behaviors among Ohio Appalachian adults. Prevention of Chronic Disease. 3(4). 127.

20. Wood, L. E., \& Bischak, G. (2000). Progress and Challenges in Reducing Economic Distress in Appalachia: An Analysis of National and Regional Trends Since 1960. Washington, DC: The Appalachian Regional Commission. 